\title{
Evaluation of humoral immunity profiles to identify heart recipients at risk for development of severe infections: a multicenter prospective study
}

\author{
Elizabeth Sarmiento MD, PhD, ${ }^{a}$ María Jaramillo LB, ${ }^{a}$ Leticia Calahorra LB, ${ }^{a}$ Juan \\ Fernández-Yáñez MD, ${ }^{\mathrm{b}}$ Miguel Gómez-Sánchez MD, PhD, ${ }^{\mathrm{c}}$ María G. Crespo-Leiro \\ MD, PhD ${ }^{\mathrm{d}}$ María Paniagua MD, PhD,${ }^{\mathrm{d}}$ Luis Almenar MD, PhD,${ }^{\mathrm{e}}$ Mónica Cebrián MS, \\ $\mathrm{PhD},{ }^{\mathrm{e}}$ Gregorio Rábago $\mathrm{MD}, \mathrm{PhD},{ }^{\mathrm{f}}$ Beltrán Levy $\mathrm{MD}, \mathrm{PhD},{ }^{\mathrm{f}}$ Javier Segovia $\mathrm{MD}, \mathrm{PhD},{ }^{\mathrm{g}}$ \\ Manuel Gómez-Bueno MD, PhD, ${ }^{\mathrm{g}}$ Javier López MD, PhD, ${ }^{\mathrm{h}}$ Sonia Mirabet MD, PhD, ${ }^{\mathrm{i}}$ \\ Joaquin Navarro LB, PhD, ${ }^{\mathrm{a}}$ Juan José Rodríguez-Molina LB, ${ }^{\mathrm{a}}$ Eduardo Fernández-Cruz \\ $\mathrm{MD}, \mathrm{PhD},{ }^{\mathrm{a}}$ Javier Carbone MD, $\mathrm{PhD}^{\mathrm{a}}$
}

\footnotetext{
${ }^{a}$ Clinical Immunology Department, Hospital General Universitario Gregorio Marañón, Madrid, Spain; ${ }^{b}$ Cardiology Department, Hospital General Universitario Gregorio Marañón, Madrid, Spain; ${ }^{c}$ Cardiology Department, Hospital Universitario Doce de Octubre, Madrid, Spain; ${ }^{d}$ Cardiology Department, Complexo Hospitalario Universitario de A Coruña, A Coruña, Spain; ' Heart Failure and Heart Transplant Unit, Cardiology Department, Hospital Universitario La Fe, Valencia, Spain; ${ }^{f}$ Heart Surgery Department, Clínica Universidad de Navarra, Pamplona, Spain; ${ }^{g}$ Heart Failure and Cardiomyopathy Unit, Heart Failure and Heart Transplant Section, Department of Cardiology, Hospital Universitario Puerta de Hierro, Madrid, Spain; ${ }^{h}$ Heart Failure and Heart Transplant Unit, Hospital Clínico Universitario, Valladolid, Spain; ${ }^{i}$ Cardiology Department, Sant Pau Hospital, Barcelona, Spain
}

Background. New biomarkers are necessary to improve detection of the risk of infection in heart transplantation. We performed a multicenter study to evaluate humoral immunity profiles that could better enable us to identify heart recipients at risk of severe infections.

Methods. We prospectively analyzed 170 adult heart recipients at 8 centers in Spain. Study points were before transplantation and 7 and 30 days after transplantation. Immune parameters included IgG, IgM, IgA and complement factors $\mathrm{C} 3$ and $\mathrm{C} 4$, and titers of specific antibody to pneumococcal polysaccharide antigens (anti-PPS) and to cytomegalovirus (CMV). To evaluate potential immunologic mechanisms leading to IgG hypogammaglobulinemia, before heart transplantation we assessed serum B-cell activating factor (BAFF) levels using enzyme-linked immunoassay. The clinical follow-up period lasted 6 months. Clinical outcome was need for intravenous antimicrobials for therapy of infection.

Results. During follow-up, 53 patients $(31.2 \%)$ developed at least 1 severe infection. We confirmed that $\operatorname{IgG}$ hypogammaglobulinemia at Day 7 (defined as $\mathrm{IgG}<600 \mathrm{mg} / \mathrm{dl}$ ) is a risk factor for infection in general, bacterial infections in particular, and CMV disease. At Day 7 after transplantation, the combination of $\mathrm{IgG}<600 \mathrm{mg} / \mathrm{dl}+\mathrm{C} 3$ $<80 \mathrm{mg} / \mathrm{dl}$ was more strongly associated with the outcome (adjusted odds ratio 7.40; 95\% confidence interval 1.48 to $37.03 ; p=0.014)$. We found that quantification of anti-CMV antibody titers and lower anti-PPS antibody concentrations were independent predictors of CMV disease and bacterial infections, respectively. Higher pretransplant BAFF levels were a risk factor of acute cellular rejection.

Conclusion. Early immunologic monitoring of humoral immunity profiles proved useful for the identification of heart recipients who are at risk of severe infection.

Keywords: complement; heart transplantation; hypogammaglobulinemia; infection; risk factors 
Infections continue to be a barrier to long-term survival in heart recipients. According to the 2014 report of the Registry of the International Society for Heart and Lung Transplantation, infection is the leading cause of death between 1 month and 1 year after transplantation. ${ }^{1}$ New early biomarkers are needed to improve assessment of the risk of developing infection after heart transplantation. ${ }^{2}$

Immunoglobulin $\mathrm{G}$ (IgG) hypogammaglobulinemia has been evaluated extensively in heart transplantation. Previous studies have analyzed the role of monitoring IgG concentrations in various immunosuppressive settings. $^{3-6}$ A recently published meta-analysis suggested that $\operatorname{IgG}$ hypogammaglobulinemia is a risk factor for infection in solid-organ recipients. ${ }^{7}$ However, previous studies are limited by the fact that data were gathered from a single institution. Multicenter studies are necessary to validate biomarkers for use in clinical practice.

In this study we present the results of a multicenter, prospective, observational study of humoral immunity biomarkers in adult heart recipients. The specific aims of this multicenter study were as follows: (1) to confirm whether IgG hypogammaglobulinemia is an independent risk factor of severe infection; (2) to identify humoral immunity profiles that could better enable us to identify the risk of infection; (3) to evaluate the reproducibility of $\mathrm{IgG}$ and complement factor 3 (C3) testing results in different centers; and (4) to evaluate potential immune factors of hypogammaglobulinemia before heart transplantation.

\section{Methods}

\section{Study design}

We prospectively evaluated 170 adult patients undergoing heart transplantation in 8 geographically dispersed teaching hospitals in Spain. The centers were distributed as follows: Madrid (Hospital General Universitario Gregorio Marañon [coordinating center, $n=59$ ], Hospital Universitario Doce de Octubre [ $n$ $=27$ ], Hospital Universitario Puerta de Hierro [ $n=8]$ ); La Coruña (Complexo Hospitalario Universitario A Coruña [ $n=26]$ ); Navarra (Clinica Universitaria de Navarra [ $n=19]$ ); Valladolid (Hospital Clínico Universitario [ $n=9]$ ); Valencia (Hospital Universitario La Fe $[n=20]$ ); and Barcelona (Hospital de la Santa Creu i Sant Pau $[n=2])$. Data were collected later at some centers than at others. Twenty-five patients from the coordinating center overlapped with the population studied in a previous publication. ${ }^{8}$

The clinical characteristics of the patients are presented in Supplementary Table S1 (available online www.jhltonline.org). Induction therapy was taken by 147 patients (86.5\%; anti-CD25 monoclonal antibodies, $n=146$; anti-thymocyte globulin, $n=1)$. The frequency of induction therapy was similar between institutions and ranged from $86 \%$ to $100 \%$. With respect to maintenance immunosuppression therapy, 54 patients $(31.8 \%)$ received cyclosporine and 107 received tacrolimus $(62.9 \%)$. Differences were observed between the centers. The frequency of administration of cyclosporine varied from $15 \%$ to $100 \%$, and that of tacrolimus from $25 \%$ to $100 \%$. Mycophenolate mofetil was taken by 164 patients (96.5\%), with few differences in frequency (range 86\% to 100\%). During the 6 months of follow-up, none of the patients used proliferation signal inhibitors (everolimus or rapamycin).

Anti-microbial prophylaxis included peri-transplant cephalosporins in 133 patients (78.2\%), quinolones during the first month in 75 (44.1\%), and trimethoprim-sulfamethoxazole during the 6-month follow-up period in 123 patients (72.4\%). As for anti-cytomegalovirus (anti-CMV) prophylaxis, 69 patients $(40.6 \%)$ received valganciclovir, 30 (17.6\%) received ganciclovir, and $47(27.6 \%)$ did not receive CMV prophylaxis. Differences were detected between the centers. The frequency of administration of valganciclovir varied from $4.2 \%$ to $100 \%$, and that of ganciclovir from $0 \%$ to $100 \%$. As for anti-fungal prophylaxis, nystatin was indicated in 147 patients $(86.5 \%)$. None of the patients received intravenous immunoglobulin (IVIg) before the immunologic tests performed at Day 7 after transplantation. One patient received IVIg after diagnosis of thrombocytopenic purpura at Day 14 after transplantation. No patients received IVIg before development of a severe infection. 
The follow-up period was the 6 months after transplantation. Severe infections were defined according to the definitions of the U.S. Centers for Disease Control and Prevention. The only infections included were severe infections, that is, those requiring intravenous anti-microbial therapy in hospital. Superficial surgical-site infections and catheter-associated infections were not included. CMV disease was defined as positive results in CMV antigenemia or polymerase chain reaction (PCR) assay and clinical symptoms.

Clinical and epidemiologic data on all patients enrolled in the study were collected by local investigators during the follow-up period. Rejection episodes were diagnosed based on endomyo-cardial biopsy findings. We included only treated rejection episodes in the analysis.

\section{Immunologic studies}

Immunologic tests were performed in serum samples obtained at inclusion on the waiting list and at Days 7 and 30after transplantation. Levels of immunoglobulins (IgG, IgA, IgM) and complement factors (C3 and C4) were determined using nephelometry (Beckman-Coulter-Izasa, Brea, CA). Variations in the kinetics and values of selected immunologic parameters (IgG and C3) among patients in heart transplant units at the various institutions were observed to assess the reproducibility of biomarkers.

The evaluation of specific risk factors of CMV disease included assessment of IgG anti-CMV antibodies using a commercial enzyme-linked immunoassay (ELISA) kit. Microtitration plates were coated with inactivated CMV antigens derived from human fibroblasts infected with CMV (Enzygnost Anti-CMV IgG; Siemens Healthcare Diagnostics, Marburg, Germany).

A substudy was also performed to identify humoral immunity-specific risk factors for severe bacterial infection. The specific IgG response to polysaccharide antigens was evaluated using a multivalent pneumococcal polysaccharide ELISA kit containing 23 serotypes of Pneumococcus pneumoniae (Binding Site; Birmingham, UK). This is an accepted surrogate biomarker for the evaluation of immune competence against polysaccharide antigens in bacterial infections.

To evaluate potential immunologic mechanisms leading to IgG hypogammaglobulinemia before heart transplantation we assessed serum B-cell activating factor (BAFF) levels in frozen samples. BAFF is a cytokine that regulates survival and selection of peripheral B-cells. Serum BAFF concentrations were assessed using a commercial ELISA test (HumanBAFF/BLyS/TNFSF13B Quantikine Test; R\&D Systems, Inc., Minneapolis, MN).

\section{Statistical analysis}

Markers were defined according to established cut-off definitions when available (reference ranges: $\mathrm{IgG}, 600$ to $1,600 \mathrm{mg} / \mathrm{dl} ; \mathrm{C} 3,80$ to $160 \mathrm{mg} / \mathrm{dl} ; \mathrm{C} 4,20$ to $40 \mathrm{mg} / \mathrm{dl}$ ), and receiver-operating characteristic (ROC) curve analysis was used to identify the best cut-off points when normal ranges were not available.

We analyzed patient-related variables (demographic, pre-operative, intra-operative and posttransplant) and 7 immunologic variables. Independent risk factors for severe infection in general and for specific infections (CMV disease or bacterial infection) were assessed using multivariable logistic regression. The odds ratio $(\mathrm{OR})$ was used as a measure of relative risk. $p<0.05$ was considered significant. A step wise approach was used to determine which of the factors or variables studied were most strongly associated with severe infections. Variables with significant colinearity (correlation coefficients $>80 \%$ ) were dropped from the analysis. Regardless of statistical significance, the variable "participating center" was left in models to account for potential unmeasured differences between the institutions in the risk of developing severe infections. A Kaplan-Meier analysis was used to calculate the cumulative probability of severe infections after transplantation according to the levels of immunologic biomarkers performed at the earliest time-point after transplantation (i.e., 7 days after transplantation). An immunologic score was then created. ORs were used to determine the number of points assigned to each 
of the earliest immunologic individual risk factors with the highest specificity $(>80 \%)$ and the sum was the score.

\section{Assessment of reproducibility}

Seven participating centers submitted serum samples to the coordinating center in Madrid to be analyzed for measurement of the selected biomarkers. Each institution received instructions on the standard procedures for sample collection, storage and shipment. All samples were aliquoted into batches of $500 \mu \mathrm{l}$. Samples were stored frozen at $-30^{\circ} \mathrm{C}$ until being used in the study. Four centers participated in the reproducibility study and tested their serum samples at their own laboratories. Data from these sites were then sent to the coordinating center. The goal of this study was to assess agreement between laboratories to evaluate the reproducibility of $\operatorname{IgG}$ and $\mathrm{C} 3$ testing. The correlation between biomarker levels tested at the coordinating and collaborating centers was assessed using Pearson's correlation test.

Analyses were performed using SPSS Statistics version 22 (IBM SPSS, Armonk, NY).

\section{Ethics}

The procedures followed in this study were in accordance with the ethical standards of our hospital ethics committee and with the 1975 Declaration of Helsinki. Before initiation of this study, institutional review board approval was obtained from the ethics committee at Gregorio Marañon Hospital (Projects FIS 050839, FIS 081430 and FIS 1101323). Local ethics committee approval was then obtained from all collaborating centers. Informed consent was obtained before the studies were performed.

\section{Results}

\section{Kinetics of biomarkers}

The rates of immunologic abnormalities in the cohort of heart recipients are presented in Supplementary Table S2 (available at www.jhlt.org). A significant decrease in IgG concentration was observed up to 1 month after transplantation. We demonstrated that the kinetics of biomarkers was similar in all participating centers (Figure 1). The complete list of centers is shown in Figure S1 (see Supplementary Material online). The kinetics of C3 differed. A significant decrease was observed at 7 days after transplantation. However, at 1 month, a recovery was observed at all centers compared with pre-transplant values (Figure 1, and Supplementary Figure S1, available at www. jhlt.org). As for specific antibodies, IgG anti-CMV and anti-pneumococcal polysaccharide antibody titers decreased significantly up to 1 month after transplantation (Figure 2). 
Center 1
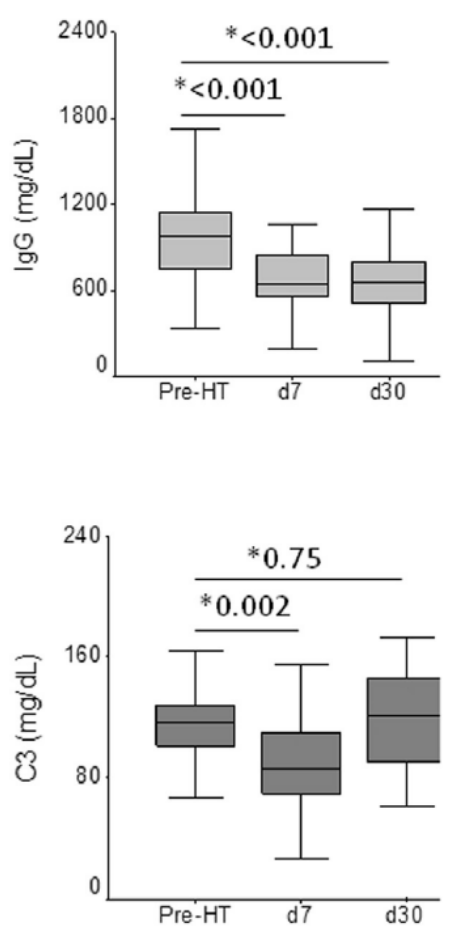

Center 2
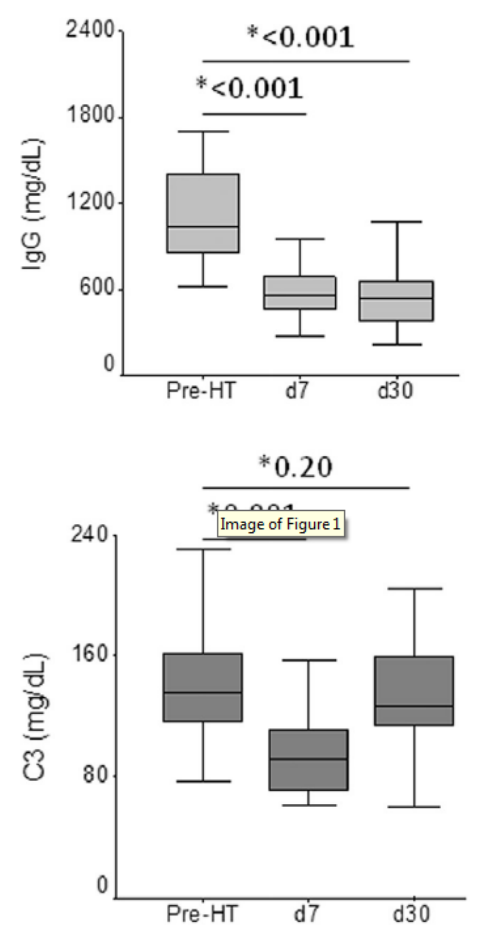

Center 6
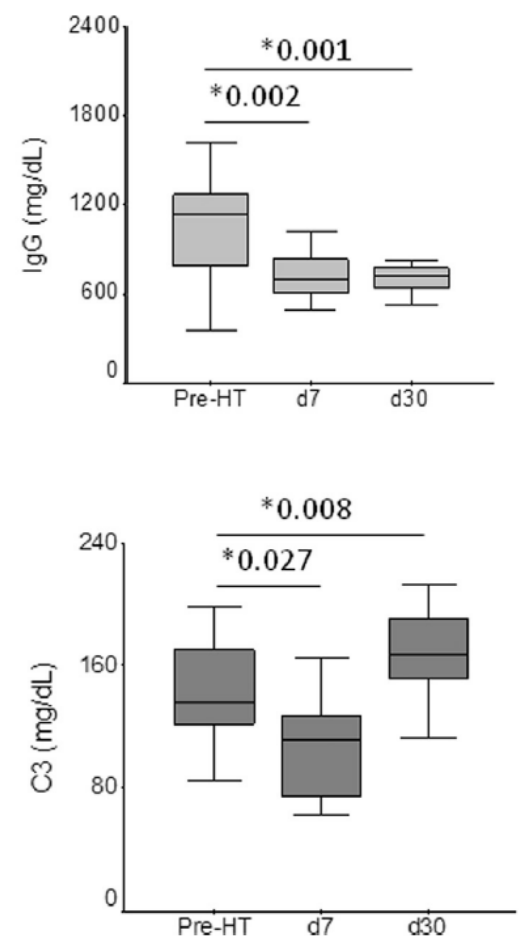

Figure 1 Kinetics of serum IgG and C3 in 3 heart transplant centers.
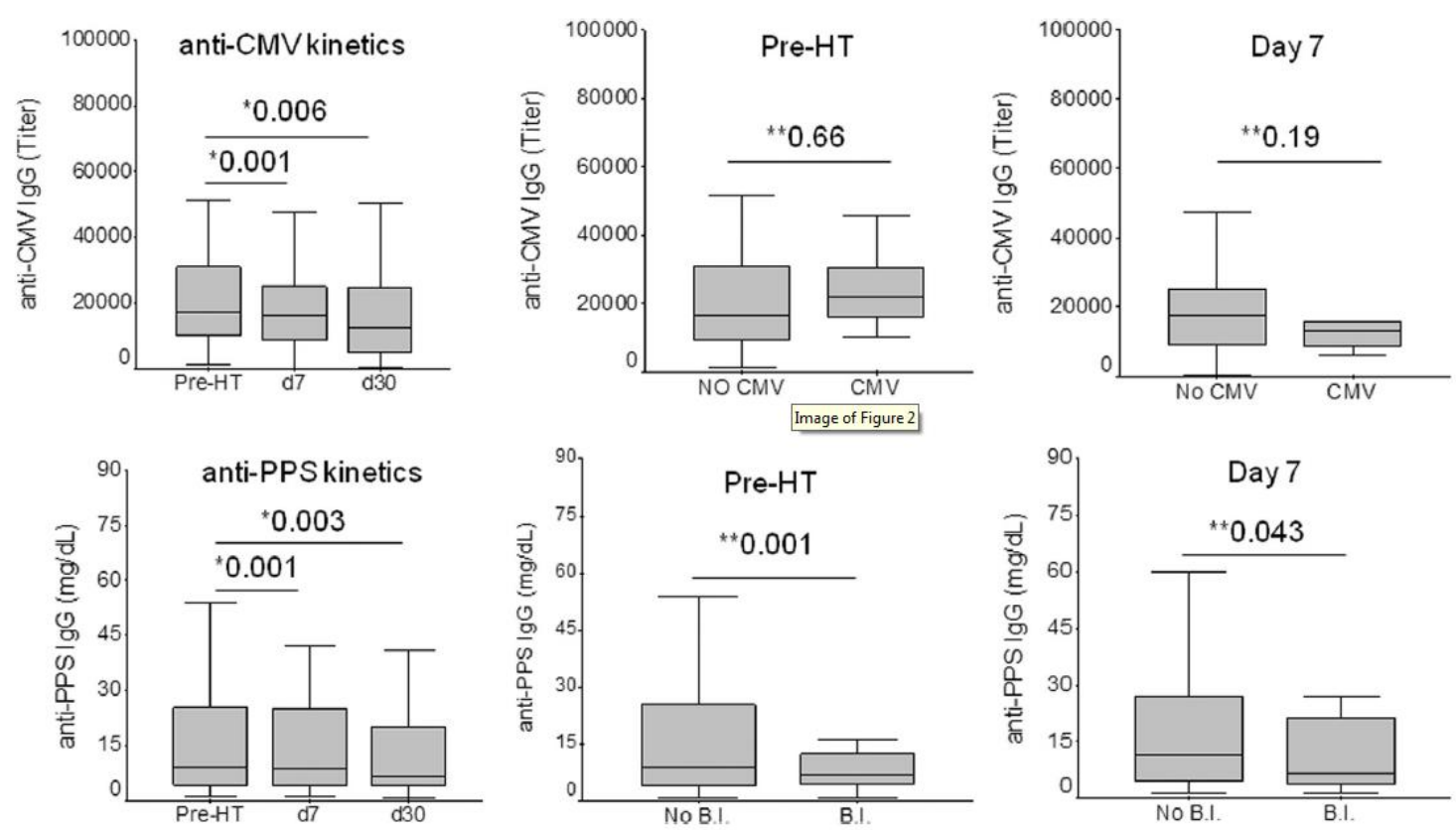

Figure 2 Kinetics of specific anti-CMVand anti-PPS antibodies. Levels in infected and non-infected patients. 


\section{Clinical risk factors for severe infection}

During the study period, 53 patients $(31.2 \%)$ developed at least 1 episode of severe infection after transplantation. Mean time to the first infection was 65 days (median 45 days, interval 2 to 180 days). Most severe infections (77\%) occurred within the first 3 months after transplantation. The distribution of microorganisms detected in the first infectious episode is presented in Supplementary Table S3 (available at www.jhlt.org). Stay at a particular institution was not significantly associated with an increased risk of severe infection (Table1).

Table 1 Clinical and Immunologic Risk Factors for Development of Severe Infection

\begin{tabular}{|c|c|c|c|c|c|}
\hline Variable & $\mathrm{Sn}$ & $\mathrm{Sp}$ & Odds ratio $^{\mathrm{b}}$ & $95 \% \mathrm{CI}$ & $p$-value \\
\hline Participating center & - & - & 0.91 & 0.79 to 1.04 & 0.17 \\
\hline Age $>50$ years $^{\mathrm{a}}$ & 87.5 & 29.7 & 3.30 & 1.19 to 9.18 & $0.022^{\mathrm{c}}$ \\
\hline Gender: male vs female & - & - & 1.57 & 0.71 to 3.44 & 0.26 \\
\hline Pre-HT diabetes mellitus & 38.8 & 78 & 2.21 & 0.95 to 5.13 & 0.065 \\
\hline Pre-HT renal failure (creatinine $>2.5 \mathrm{mg} / \mathrm{dl}$ ) & - & - & 1.27 & 0.43 to 3.79 & 0.66 \\
\hline Positive Mantoux result & - & - & 0.99 & 0.458 to 2.184 & 0.98 \\
\hline Pre transplant hypoalbuminemia $(<3.4 \mathrm{~g} / \mathrm{dl})$ & - & - & 1.72 & 0.61 to 4.83 & 0.30 \\
\hline \multicolumn{6}{|l|}{ Donor risk factors } \\
\hline Donor gender: male vs female & - & - & 0.99 & 0.47 to 2.06 & 0.97 \\
\hline Donor age 450 years $^{\mathrm{a}}$ & - & - & 0.67 & 0.17 to 2.70 & 0.57 \\
\hline Discordant CMV receptor negative/donor positive & 12 & 96 & 3.45 & 0.92 to 12.85 & 0.07 \\
\hline Ventricular assist device & - & - & 1.44 & 0.63 to 3.27 & 0.38 \\
\hline 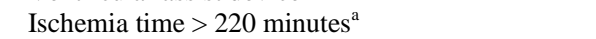 & - & - & 1.47 & 0.69 to 3.09 & 0.31 \\
\hline Extracorporeal circulation time $>127$ minutes $^{\mathrm{a}}$ & 61 & 55 & 2.01 & 0.94 to 4.29 & 0.07 \\
\hline Intubation time $>14$ hours $^{\mathrm{a}}$ & 69 & 64 & 3.87 & 1.77 to 8.48 & $0.0007^{\mathrm{c}}$ \\
\hline Time inintensive care unit $>6$ days $^{\mathrm{a}}$ & 67 & 63 & 3.55 & 1.64 to 7.67 & $0.0013^{\mathrm{c}}$ \\
\hline Induction: basiliximab vs daclizumab & - & - & 0.74 & 0.30 to 1.81 & 0.51 \\
\hline Induction: methylprednisolone & - & - & 0.84 & 0.36 to 1.96 & 0.68 \\
\hline Maintenance: cyclosporine & 74.5 & 56 & 0.42 & 0.19 to 0.93 & $0.032^{\mathrm{c}}$ \\
\hline Maintenance: tacrolimus & - & - & 1.76 & 0.84 to 3.71 & 0.13 \\
\hline Use of anti-CMV prophylaxis (yes vs no) & - & - & 1.44 & 0.58 to 3.43 & 0.44 \\
\hline \multicolumn{6}{|l|}{ Immunologic risk factors } \\
\hline Day 7 C4 hypocomplementemia $(<20 \mathrm{mg} / \mathrm{dl})$ & 58 & 64 & 1.98 & 1.03 to 5.05 & $0.045^{\mathrm{c}}$ \\
\hline Day $30 \mathrm{IgG}<450 \mathrm{mg} / \mathrm{dl}^{\mathrm{a}}$ & 23 & 91 & 3.24 & 1.14 to 9.19 & $0.027^{\mathrm{c}}$ \\
\hline Day $30 \mathrm{IgM}<50 \mathrm{mg} / \mathrm{dl}^{\mathrm{a}}$ & 38 & 82 & 2.62 & 1.08 to 6.32 & $0.033^{\mathrm{c}}$ \\
\hline Day $30 \mathrm{C} 3$ hypocomplementemia $\left(<90 \mathrm{mg} / \mathrm{dl}^{\mathrm{a}}\right)$ & 19 & 93 & 3.14 & 1.01 to 9.73 & $0.047^{\mathrm{c}}$ \\
\hline Day $7 \mathrm{IgG}<600 \mathrm{mg} / \mathrm{dl}$ and C3 $<80 \mathrm{mg} / \mathrm{dl}$ & 30 & 93 & 5.52 & 1.57 to 19.38 & $0.007^{\mathrm{c}}$ \\
\hline
\end{tabular}

C3, complement factor 3; CI, confidence interval; HT, heart transplantation; IgG, immunoglobulin G; Sn, sensitivity; Sp, specificity.

${ }^{a}$ Cut-off was established by receiver-operating characteristic analysis.

${ }^{\mathrm{b}}$ Bivariate analysis after adjustment for participating center.

${ }^{c}$ Significant risk factors for development of severe infection. 
The risk factors for development of severe infection are presented in Table 1. Patients > 50 years of age were at higher risk of severe infection. Intubation time and time in the intensive care unit after transplantation were also significant clinical risk factors for infection. There was a trend toward increased risk of infection among patients who had diabetes, CMV-negative serostatus in the case of patients who had received a transplant from a CMV-positive donor, and in patients with a longer extracorporeal circulation time. As for the association between medication and increased risk of developing severe infection, treatment with tacrolimus tended to be a risk factor. Pre-transplant concentrations of total proteins and albumin were similar in both groups (infected vs uninfected patients; see Supplementary Table S1). Pre-transplant hypoalbuminemia, infection before transplant, previous mechanical ventilation and high-urgency waiting list status were not associated with risk of infection (Table1).

We analyzed the impact of anti-microbial prophylaxis on the rate of severe infections in the early post-operative period (14 days after transplantation, $n=14)$. We found no association between cephalosporins and development of early infections $(\mathrm{OR}=0.69,95 \%$ confidence interval $[\mathrm{CI}] 0.18$ to 2.67, $p=0.59$ ). When we evaluated the relationship between therapy with quinolones during the first month and the rate of early infection, again, this variable was not significantly associated with early infection $(\mathrm{OR}=2.28,95 \%$ CI 0.55 to $9.49, p=0.26)$.

\section{Immunologic risk factors of severe infection}

An immunologic study was performed at inclusion on the waiting list. Patients with $\operatorname{IgG}$ hypogammaglobulinemia (defined as $\mathrm{IgG}<600 \mathrm{mg} / \mathrm{dl}$ ) and patients with lower $\mathrm{C} 3$ levels were at higher risk of infection. One week after transplantation, IgG hypogammaglobulinemia, C3 hypocomplementemia (defined as C3 $<80 \mathrm{mg} / \mathrm{dl}$ ) and C4 hypocomplementemia (defined as C4 $<20 \mathrm{mg} / \mathrm{dl}$ ) were significant risk factors for severe infection (Table 1). At Day 30 after transplantation, a lower IgG cut-off $(<450$ $\mathrm{mg} / \mathrm{dl}$ ) established by ROC curve analysis was associated with risk of infection, as were lower IgM and C3 levels (Table 1).

\section{Multivariate analyses}

Table 2 shows the results of the multivariate analysis for the entire cohort with respect to the immunologic factors that were independently associated with increased risk of severe infection after adjustment for age > 50 years, number of days in the ICU, use of cyclosporine and participating center. The variable intubation time was not included in the final model because it was significantly associated with time in the ICU (2-tailed correlation, $p<0.0001$ ). IgG hypogammaglobulinemia at Days 7 and 30 and C3 hypocomplementemia at Day 7 continued to be significant risk factors.

Interestingly, the combination of both factors at Day 7 was more strongly associated with the risk of severe infection (Table 2).

Figure 3 shows the Kaplan-Meier survival curves for length of time after immunologic monitoring at Day 7 until occurrence of severe infection for heart recipients, according to levels of the independent immunologic predictors. 
Table 2 Multivariate Logistic Regression Analysis of Immunologic Risk Factors for Development of Severe Infection

\begin{tabular}{|c|c|c|c|}
\hline Variable & Odds ratio ${ }^{\mathrm{b}}$ & $95 \% \mathrm{CI}$ & $p$-value \\
\hline Pre-HT IgG hypogammaglobulinemia (< 600 mg/dl) & 1.88 & 0.54 to 6.14 & 0.325 \\
\hline Pre-HT C $3<126 \mathrm{mg} / \mathrm{dl}^{\mathrm{a}}$ & 1.56 & 0.61 to 4.01 & 0.351 \\
\hline Day 7 IgG hypogammaglobulinemia $(<600 \mathrm{mg} / \mathrm{dl})$ & 4.39 & 1.32 to 14.61 & 0.015 \\
\hline Day $7 \mathrm{C} 3$ hypocomplementemia $(<80 \mathrm{mg} / \mathrm{dl})$ & 5.81 & 1.28 to 26.40 & 0.022 \\
\hline Day 7 C4 hypocomplementemia (<20 mg/dl) & 1.09 & 0.31 to 3.90 & 0.88 \\
\hline Day $30 \mathrm{C} 3$ hypocomplementemia $\left(<90 \mathrm{mg} / \mathrm{dl}^{\mathrm{a}}\right)$ & 1.66 & 0.43 to 6.41 & 0.46 \\
\hline Profile Day7: $\operatorname{IgG}<600 \mathrm{mg} / \mathrm{dl}$ and $\mathrm{C} 3<80 \mathrm{mg} / \mathrm{dl}$ & 7.40 & 1.48 to 37.03 & 0.014 \\
\hline Immunologic score $\geq 4$ & 11.0 & 2.68 to 45.07 & 0.0009 \\
\hline
\end{tabular}

C3, complement factor 3; CI, confidence interval; HT, heart transplantation; IgG, immunoglobulin G.

${ }^{\text {a }}$ Cut-off was established by receiver-operating characteristic analysis.

${ }^{\mathrm{b}}$ After adjustment for treatment with cyclosporine, age $>50$ years, time in the intensive care unit $>6$ days, and participating center. Odds ratios were used to determine the number of points assigned to each of the 4 individual risk factors of the immunologic score (pre-transplant $\mathrm{IgG}<600 \mathrm{mg} / \mathrm{dl}=2$ points, discordant $\mathrm{CMV}$ receptor negative/donor positive $=3$ points, Day $7 \mathrm{IgG}<600 \mathrm{mg} / \mathrm{dl}=$ 2 points and Day $7 \mathrm{C} 3<80 \mathrm{mg} / \mathrm{dl}=5$ points $)$.
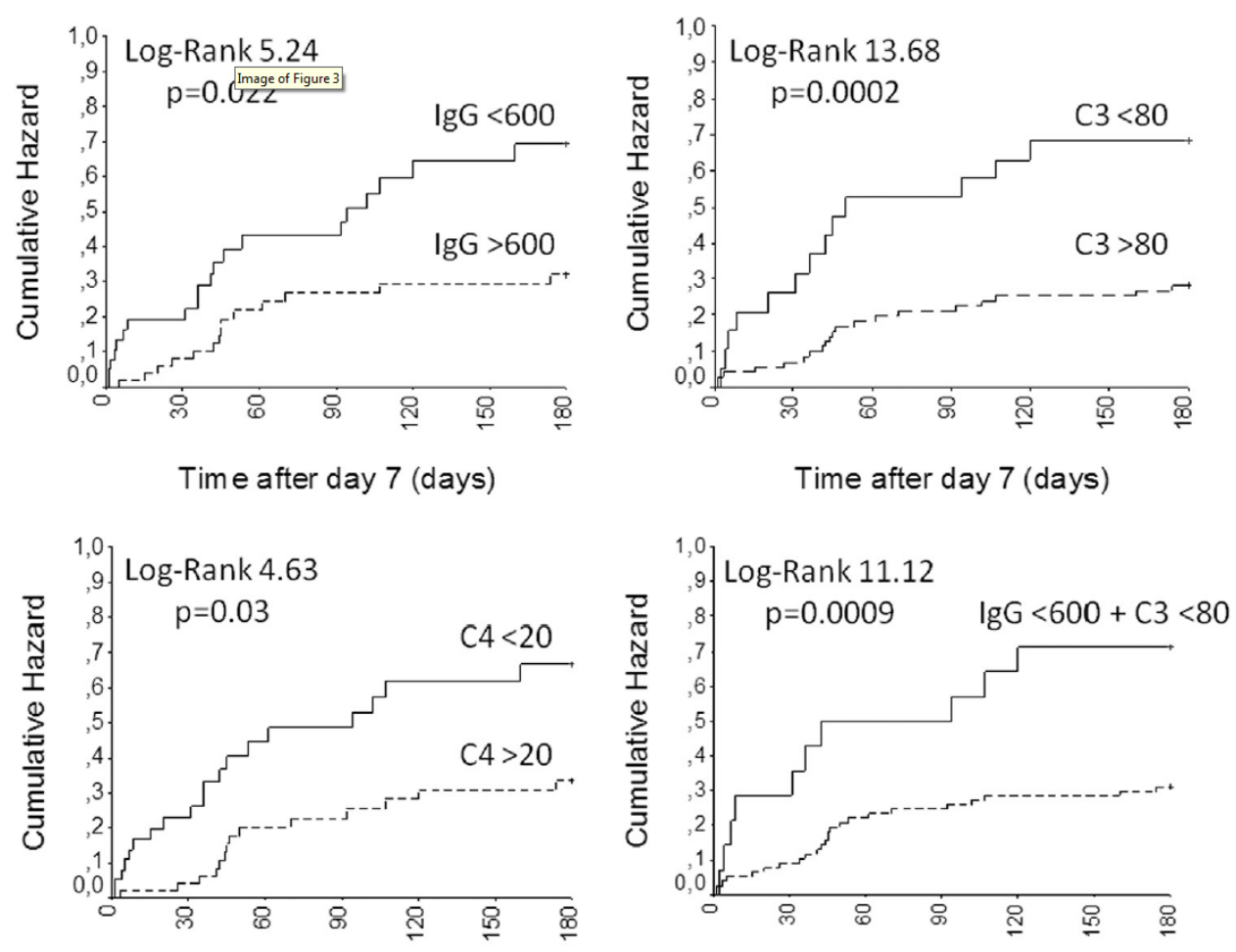

Figure 3 Kaplan-Meier curves showing the cumulative risk for development of severe infection during follow-up according to levels of distinct humoral immunity biomarkers performed at 7 days after transplantation. IgG: $\operatorname{serum~IgG(mg/dl);~C3:~serum~}$ complement C3 (mg/dl); C4: serum complement C4 (mg/dl). 


\section{Immunologic score}

An immunologic score was then created. Odds ratios were used to determine the number of points assigned to each of the earliest individual risk factors (assessed before transplantation and at Day 7) with the highest specificity ( $>80 \%$; pre-transplant $\mathrm{IgG}<600 \mathrm{mg} / \mathrm{dl}=2$ points, discordant CMV receptor negative/donor positive $=3$ points, Day $7 \mathrm{IgG}<600 \mathrm{mg} / \mathrm{dl}=2$ points, Day $7 \mathrm{C} 3<80 \mathrm{mg} / \mathrm{dl}=5$ points). ROC analysis showed maximum efficiency at a score of $\geq 4$ to be a cut-off for severe infection. After adjustment for clinical risk factors, an immunologic score of $\geq 4$ indicated an 11-fold greater risk for developing severe infection during the first 6 months after transplantation (Table2). The score effect with the risk of severe infection was as follows: severe infection developed in $15.6 \%$ of patients with 0 point; $32.2 \%$ of patients with 1 to 3 points; and $61.1 \%$ of patients with $\geq 4$ points (2-sided chi-square test, $p=$ $0.003)$.

\section{Immunologic risk factors for specific infections}

For this analysis, we considered the prevalence of the first specific infection detected. A total of 33 patients had severe bacterial infections. Univariate regression analysis revealed the immunologic risk factors for development of severe bacterial infections to be IgG hypogammaglobulinemia $(<600 \mathrm{mg} / \mathrm{dl})$, C3 and C4 hypocomplementemia at Day 7 and $\mathrm{IgG}$ levels $<500 \mathrm{mg} / \mathrm{dl}$ and C3 and C4 hypocomplementemia at 1 month (see Table S4 in Supplementary Material online). The independent risk factors for severe bacterial infection after adjustment for clinical risk factors were $\mathrm{IgG}$ hypogammaglobulinemia and C3 hypocomplementemia at Day 7 and $\mathrm{IgG}$ levels $<500 \mathrm{mg} / \mathrm{dl}$ at 1 month (Table 3).

Table 3 Multivariate Logistic Regression Analysis of Immunologic Risk Factors for Development of Severe Bacterial Infections

\begin{tabular}{lcc}
\hline Variable & Odds ratio $^{\text {b }}$ & $95 \%$ CI \\
\hline & & $p$-value \\
Day 7 IgG hypogammaglobulinemia $(<600 \mathrm{mg} / \mathrm{dl})$ & 3.36 & 1.13 to 9.97 \\
Day 7 C3 hypocomplementemia $(<80 \mathrm{mg} / \mathrm{dl})$ & 3.59 & 1.05 to 12.24 \\
Day 30 IgG hypogammaglobulinemia $(<500 \mathrm{mg} / \mathrm{dla})$ & 1.73 & 0.55 to 5.42 \\
Day 30 C3 hypocomplementemia $(<80 \mathrm{mg} / \mathrm{dl})$ & 9.47 & 1.14 to 78.77 \\
Profile anti-PPS $<5$ mg at Day 7 and 30 after HT $\mathrm{HT}^{\mathrm{c}}$ & 14.82 & 3.44 to 63.77 \\
Profile Day 7 IgG $<600 \mathrm{mg} / \mathrm{dl}$ and C3 $<80 \mathrm{mg} / \mathrm{dl}$ & 12.37 & 3.38 to 45.28 \\
& & 0.35 \\
\hline
\end{tabular}

C3, complement factor 3; CI, confidence interval; IgG, immunoglobulin G; PPS, polysaccharide antigen. ${ }^{\text {a }}$ Cut-off was established by receiver-operating characteristic analysis.

${ }^{\mathrm{b}}$ After adjustment for the following clinical variables: time in intensive care unit $>6$ days and participating center.

c After adjustment for the following clinical variables: time inintensive care unit > 6 days; use of pneumococcal vaccine; and participating center.

We identified 2 humoral immunity profiles that were more strongly associated with the risk of bacterial infections: presence of IgG hypogammaglobulinemia and C3 hypocomplementemia at 1 week and maintained low anti-polysaccharide response, defined as levels of anti-PPS antibodies $<5 \mathrm{mg} / \mathrm{dl}$ at Days 7 and 30 (Table 3).

CMV disease was the first infectious complication in 16 patients during the study period. Meantime from transplantation to development of CMV disease was 103 days (interval 44 to 180 days) (see Table S5 in Supplementary Material online). Independent immunologic risk factors for CMV disease after transplantation were IgG hypogammaglobulinemia and lower anti-CMV titers at Day 7, IgG levels $<500$ $\mathrm{mg} / \mathrm{dl}, \mathrm{IgA}$ levels $<80 \mathrm{mg} / \mathrm{dl}$ and C3 levels $<80 \mathrm{mg} / \mathrm{dl}$ at 1 month. Interestingly, having a lower antiCMV antibody titer was an independent risk factor after adjustment for discordant CMV serostatus as the classic risk factor for CMV disease (Table 4). 
Table 4 Multivariate Logistic Regression Analysis of Immunologic Risk Factors for Development of CMV Disease

\begin{tabular}{lcc}
\hline Variable & Odds ratio $^{\mathrm{b}}$ & 95\% CI \\
\hline & & \\
Day 7 IgG hypogammaglobulinemia $(<600 \mathrm{mg} / \mathrm{dl})$ & 6.96 & 1.34 to 36.04 \\
Day 7 anti-CMV titer $<15,000^{\mathrm{a}}$ & 8.53 & 1.57 to 46.22 \\
Day 7 C3 hypocomplementemia $(<80 \mathrm{mg} / \mathrm{dl})$ & 3.35 & 0.81 to 13.90 \\
Profile Day 7: IgG $<600 \mathrm{mg} / \mathrm{dl}$ and C3 $<80 \mathrm{mg} / \mathrm{dl}$ & 3.73 & 0.83 to 16.76 \\
Day 30 IgG hypogammaglobulinemia $\left(<500 \mathrm{mg} / \mathrm{dl}^{\mathrm{a}}\right)$ & 8.91 & 0.013 \\
Day 30 IgA hypogammaglobulinemia $\left(<80 \mathrm{mg} / \mathrm{dl}^{\mathrm{a}}\right)$ & 17.08 & 2.15 to 36.84 \\
Day 30 C3 hypocomplementemia $\left(<80 \mathrm{mg} / \mathrm{dl}^{\mathrm{a}}\right)$ & 13.26 & 0.095 \\
Discordant CMV serologic status $\left(\mathrm{D}^{+} / \mathrm{R}^{-}\right)^{\mathrm{c}}$ & 7.03 & 1.93 to 90.88 \\
& & 0.24 to 207.33 \\
\hline
\end{tabular}

$\mathrm{CI}$, confidence interval; $\mathrm{CMV}$, cytomegalovirus; $\mathrm{D}^{+}$, donor positive; $\mathrm{R}^{-}$, recipient negative.

${ }^{a}$ Cut-off was established by receiver-operating characteristic analysis.

${ }^{b}$ After adjustment for the following clinical variables: discordant CMV serologic status of D and R; methylprednisolone use in induction protocol; and participating center.

${ }^{c}$ After adjustment for the following variables: IgG Day $7<600 \mathrm{mg} / \mathrm{dl}$; methylprednisolone use in induction protocol; and participating center.

\section{Early fatal infections}

Eight patients $(4.7 \%)$ died of their infections during the first 2 weeks after transplantation. Before heart transplantation, these patients had significantly lower levels of C3 (116 $\pm 13 \mathrm{vs} 139 \pm 37 \mathrm{mg} / \mathrm{dl}, p=$ 0.006 ) and of anti-pneumococcal polysaccharide antibody ( $9 \pm 8$ vs $29 \pm 65 \mathrm{mg} / \mathrm{dl}, p=0.004)$. A trend toward lower levels of pre-transplant $\operatorname{IgA}(203 \pm 99$ vs $284 \pm 137 \mathrm{mg} / \mathrm{dl}, p=0.057)$ and C4 $(22 \pm 7$ vs 26 $\pm 9 \mathrm{mg} / \mathrm{dl}, p=0.16)$ was also observed. Four of 11 patients $(36.4 \%)$ who required previous mechanical support developed early fatal infections, as compared with only 4 of $159(2.5 \%)$ heart recipients not using ventricular assist devices $(p<0.001)$. Donor age was significantly higher in these patients $(52 \pm 4$ vs $40 \pm$ $12, p=0.032)$. Age $>50$ years $(p=0.58)$, induction therapy $(p=0.45)$, previous mechanical ventilation $(p=0.93)$, intubation time $(p=0.75)$, high urgency $(p=0.37)$, therapy with cephalosporins $(p=0.49)$ and therapy with quinolones $(p=0.25)$ were not associated with fatal infections.

\section{Variability of biomarkers between centers}

A total of $122 \mathrm{IgG}$ and $170 \mathrm{C} 3$ determinations were performed at the collaborating centers. Good reproducibility was observed for pre- and post-transplant IgG and C3 levels when these values were correlated with the values obtained after assessment of the same serum samples at the coordinating center (Figure 4). 

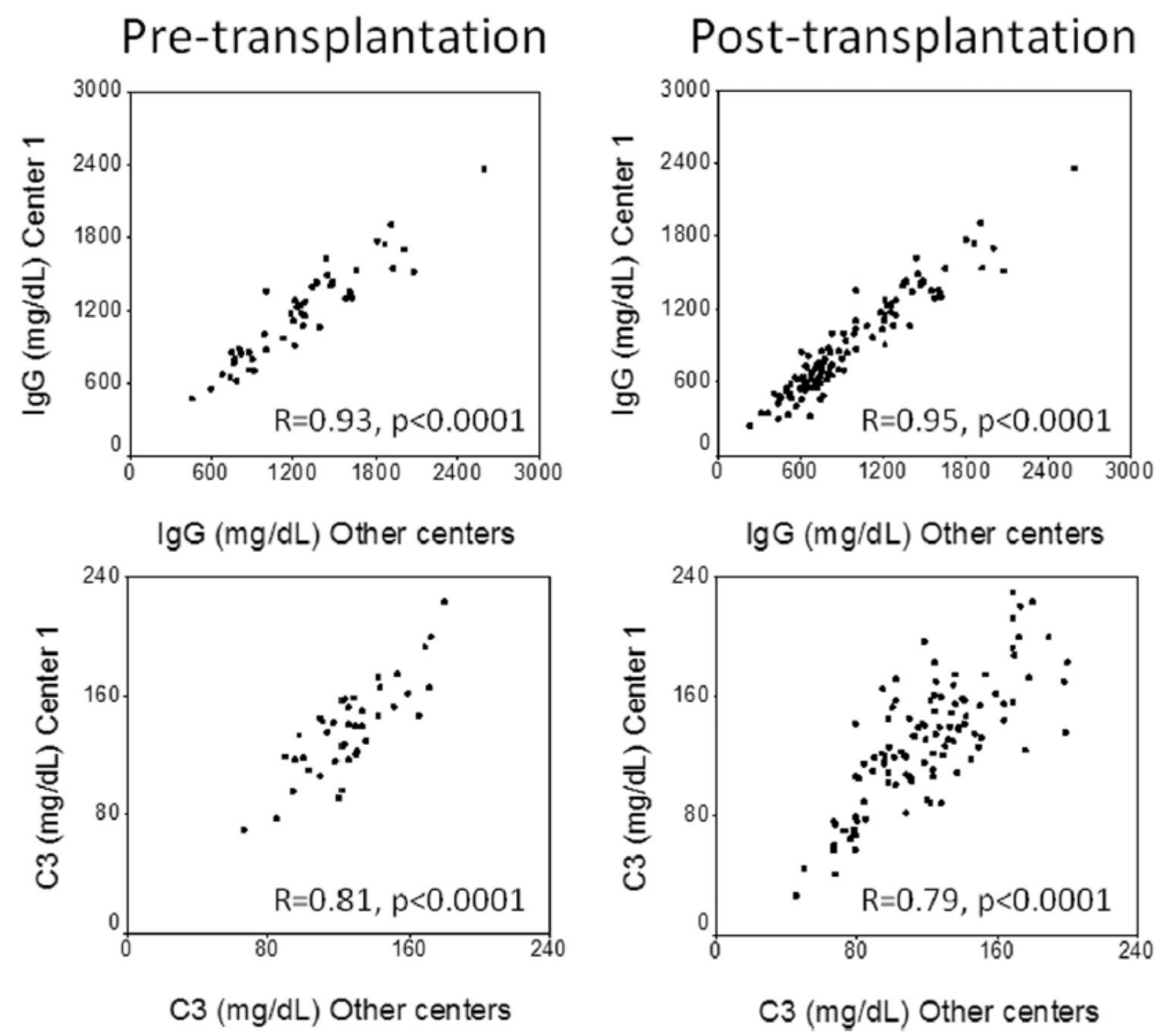

Figure 4 Evaluation of reproducibility of serum $\mathrm{IgG}$ and $\mathrm{C} 3$ testing.

Baseline serum BAFF levels, IgG hypogammaglobulinemia and clinical outcomes

Median pre-transplant serum BAFF concentration of the entire cohort was $1,366.59 \mathrm{pg} / \mathrm{ml}(388.6$ to $3,842.10 \mathrm{pg} / \mathrm{ml}$ ). BAFF levels disclosed a weakly significant correlation with IgG concentration (Pearson's correlation coefficient $0.307 ; p=0.002$ ). Median BAFF levels before heart transplantation were lower in patients with pre-transplant IgG hypogammaglobulinemia than in patients without IgG hypogammaglobulinemia, although the difference was not significant $(1,007.7$ [453.7 to 2,030.2] vs $1,415.7$ [388.6 to $3,842.1 \mathrm{pg} / \mathrm{ml}$ ]; $p=0.177$ ). When we stratified the patients according to the median value of BAFF, we found no significant correlation with IgG hypogammaglobulinemia at Day 7 (OR = $0.83,95 \%$ CI 0.31 to $2.24 ; p=0.71)$, IgG hypogammaglobulinemia at Day 30 (OR =0.86, 95\% CI 0.38 to $1.96 ; p=0.71)$ or development of post-transplant severe infections $(\mathrm{OR}=0.53,95 \% \mathrm{CI} 0.22$ to $1.26 ; p$ $=0.15)$. We also analyzed the relationship between pre- transplant BAFF levels and other clinical outcomes. During follow-up, 22\% of patients developed acute cellular rejection. Interestingly, higher serum BAFF level $(>1,400 \mathrm{pg} / \mathrm{ml})$ was a risk factor for development of acute cellular rejection $(\mathrm{OR}=$ $2.99,95 \%$ CI 1.15 to7.82; $p=0.024$ ) (Figure 5). The OR for acute cellular rejection remained significant after adjustment for the variable "participating center" (OR $=3.04,95 \%$ CI 1.15 to $8.06 ; p=0.025)$. 

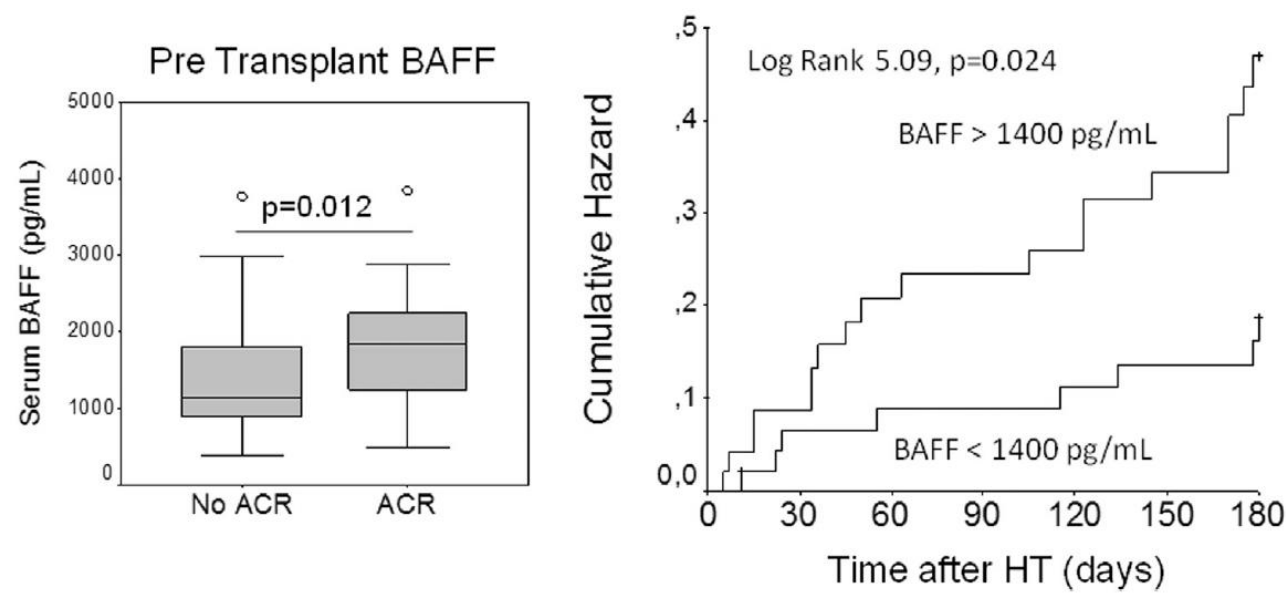

Figure 5 Pre-transplant levels of BAFF were significantly lower in heart recipients developing acute cellular rejection after transplantation. Kaplan-Meier curves show the cumulative risk for development of acute cellular rejection during follow-up after transplantation according to pre-transplant serum BAFF levels.

\section{Discussion}

No new predictors of infection in heart recipients have been incorporated into clinical practice in the last few years.

Some biomarkers are already considered risk factors for specific severe infections and are being included in guidelines, that is, measurement of specific anti-CMV cellular responses for development of CMV disease. ${ }^{9-11}$ Further validation of specific biomarkers in large-scale international multicenter studies is necessary before they can be accepted as predictors of the risk of infection in clinical practice. Unfortunately, no biomarker has been sufficiently validated to be acceptable. We have provided relevant information in this regard.

IgG hypogammaglobulinemia has been evaluated extensively as a potential biomarker in specific heart transplantation settings. ${ }^{3-7}$ However, IgG levels and other markers of humoral immunity are not included in routine protocols. We have presented the results of the first multicenter, prospective, followup study performed in adult heart recipients to assess $\operatorname{IgG}$ hypogammaglobulinemia as a predictor of severe infections.

We found that, early after transplantation (i.e.,1week), IgG hypogammaglobulinemia is an independent risk factor for severe infection in general, bacterial infections in particular, and CMV disease. We previously demonstrated that other humoral immunity markers, including C3 hypocomplementemia, are associated with a high risk of infection. ${ }^{8,12}$ We demonstrated that combined assessment of IgG hypogammaglobulinemia and C3 hypocomplementemia at 1 week after transplantation is a robust approach for identification of heart recipients at risk of infections. Importantly, we observed that serum levels of both components in this simple and easily available humoral immunity profile (IgG and C3 levels) correlated well between the coordinating center and the collaborating centers. This finding is important when considering the introduction of these biomarkers into clinical practice.

Among the markers tested for identifying the risk of specific infectious complications, the predictive value of anti-CMV titers was greater than that obtained with the classic risk factor of donor-recipient discordance in CMV serostatus. Low anti-CMV antibody titers were also associated with a high risk of CMV infection among CMV recipients with a positive serostatus (data not shown). ${ }^{13} \mathrm{~A}$ limitation of this biomarker is that it is based on an ELISA that used CMV antigensin fibroblasts. It has been suggested that the evaluation of humoral anti-CMV responses is better when performed using tests based on 
epithelialcells. ${ }^{14}$ We have previously described the potential role of this biomarker combined with the assessment of CD8 cellular responses against the IE-1 antigen of CMV to identify the risk of CMV infection. ${ }^{9}$

Low IgG anti-PPS levels at Days 7 and 30 post-transplant identified those individuals who were most likely to develop severe bacterial infections. This is a notable finding, given that severe bacterial infections are the most frequent infectious complications after heart transplantation. ${ }^{15}$ The Anti-PPS test is commonly used as a surrogate of the anti-polysaccharide immune response in patients with recurrent infections. Thus, the lower the anti-polysaccharide antibody response, the higher the risk of bacterial infections. A limitation of this evaluation is that it is affected by the type of pneumococcal vaccine used in the pre-transplant period. All patients evaluated had received 1 dose of a 23 -valent pneumococcal polysaccharide vaccine. Updated recommendations include conjugated 13-valent pneumococcal vaccine for adult transplant candidates and recipients followed by the polysaccharide vaccine to increase serotype coverage. ${ }^{16}$ New ways of evaluating the maintenance of anti- polysaccharide response after transplantation should be evaluated.

The Cleveland Clinic Group started the process of evaluating IgG hypogammaglobulinemia as a biomarker in heart transplantation. This biomarker has also been evaluated in other types of solid-organ transplantation. ${ }^{17-21}$ The results of our national multicenter study add to previous findings from a metaanalysis on the process of validating this biomarker.

In spite of its low sensitivity, a humoral immunity profile (i.e., $\mathrm{IgG}<600 \mathrm{mg} / \mathrm{dl}+\mathrm{C} 3<80 \mathrm{mg} / \mathrm{dl}$ at Day 7) may prove to be a reliable marker for monitoring the risk of severe infection in heart recipients, owing to its high specificity.

We propose an immunologic score based on humoral immunity parameters that may be useful for identifying a subgroup of heart recipients at high risk of severe infections. We previously evaluated the role of combined use of humoral and cellular biomarkers in a single-center study. ${ }^{8}$ The potential role of adenosine triphosphate (ATP) measurement on $\mathrm{CD}^{+} \mathrm{T}$ cells has also been evaluated in a longitudinal study. Kobashigawa et al ${ }^{22}$ observed that ATP levels were significantly lower among patients in whom infections were diagnosed within 1 month after evaluation than among those who did not develop infection. Because it is unlikely that any single marker predicts everything, future prospective, multicenter studies evaluating the combination of humoral immune responses with functional cellular responses are anticipated. The cost of measuring the biomarkers should be taken into account. As an example, reagents for $\operatorname{IgG}$ and $\mathrm{C} 3$ levels cost around $€ 5$ per test, whereas reagents for the functional evaluation of ATP production by CD4 cells cost about $€ 212$ per test.

To evaluate potential humoral immunity mechanisms leading to IgG hypogammaglobulinemia, we assessed serum BAFF levels in the pre-transplant evaluation. We failed to demonstrate any statistically significant association between BAFF levels and IgG hypogammaglobulinemia or severe infection. Interestingly, when evaluating the relationship between this biomarker and other clinical outcomes, we found an association with acute cellular rejection. The binding of BAFF to the BAFF receptor expressed by a subset of $\mathrm{CD} 4+\mathrm{T}$ cells stimulates T-cell activation and alloproliferation in mice. ${ }^{23} \mathrm{An}$ experimental model of heart transplantation showed that targeting the BAFF cytokine network inhibits both humoral and cellular immune responses induced by memory CD4 T cells. ${ }^{24}$ For the first time, we suggest the potential role of serum BAFF as a pre-transplant biomarker of acute cellular rejection in heart recipients.

\section{Limitations}

A limitation of our study is the inclusion of centers with a small number of patients. The Cis of some biomarkers were wide, indicating that the overall sample size was small. Binary cut-offs could not yield homogeneous populations that may continue to be present in larger samples, although the 95\% CI swould be narrower. Some of the variables used probably have more than 2 risk populations, and reducing to 2 populations produces larger 95\% CIs. We identified several clinical risk factors of infection; however, 
other major clinical risk factors for infection may not have been identified owing to the low statistical power. Nevertheless, in this proof-of-concept study, some variables were clearly associated with the incidence of infection, and the metrics of these variables could be analyzed in further studies to establish more accurate boundaries. The logical next step is an international multicenter study in this field with a larger number of patients.

\section{Disclosure statement}

The authors have no conflicts of interest to disclose. This multicenter study was supported by research grants from the Fondo de Investigacion Sanitaria (Instituto de Salud CarlosIII, Ministry of Economy and Competitiveness), Madrid, Spain (FIS 081430, FIS 1101323 and FIS 1501472, including funds from European regional development funds, "A Way of Making Europe", from the European Union). E.S. received an educational grant from Grifols (Barcelona, Spain), and holds a Joan Rodes contract from the Instituto de Salud Carlos III, Ministry of Economy and Competitiveness. J.C. received an Investigator Global Sponsored Research Grant from Grifols, Inc. (Los Angeles, CA). J.C. received are search grant from CSL Behring (Barcelona, Spain). The authors thank the heart surgery, anesthesiology, cardiology and immunology nursing teams at all the participating centers. We are also grateful to personnel of the Immunochemistry Section for quality control of the humoral immunity markers at Gregorio Marañon Hospital, Madrid (UNE-EN ISO ENAC 15189 accreditation). Thomas O'Boyle reviewed the English version of the manuscript.

\section{Supplementary materials}

Supplementary materials associated with this article can be found in the online version at www.jhltonline.org.

\section{References}

1. Lund LH, Edwards LB, Kucheryavaya AY, et al. The Registry of the International Society for Heart and Lung Transplantation: thirty-first official adult heart transplant report-2014; Focus theme: retransplantation. J Heart Lung Transplant 2014;33:996-1008.

2. Carbone J, Lanio N, Gallego A, et al. Immune monitoring to predict the development of infections after immune suppression for solid organ transplantation and autoimmune diseases. Current Drug Safety 2008;3:91-99.

3. Corales R, Chua J, Mawhorter S, et al. Significant post-transplant hypogammaglobulinemia in six heart transplant recipients: an emerging clinical phenomenon? Transpl Infect Dis 2000;2:133-9.

4. Yamani MH, Avery RK, Mawhorter SD, et al. Hypogammaglobulinemia following cardiac transplantation: a link between rejection and infection. J Heart Lung Transplant 2001;20:425-30.

5. Sarmiento E, Rodríguez-Molina J, Muñoz P, et al. Decreased levels of serum immunoglobulins as a risk factor for infection after heart transplantation. Transplant Proc 2005;37:4046-9.

6. Sarmiento E, Rodriguez-Molina JJ, Fernandez-Yañez J, et al. IgG monitoring to identify the risk for development of infection in heart transplant recipients. Transpl Infect Dis 2006;8:49-53.

7. Florescu DF, Kalil AC, Qiu F, et al. What is the impact of hypogammaglobulinemia on the rate of infections and survival in solid organ transplantation? A meta-analysis. Am J Transplant 2013;13:260110 .

8. Sarmiento E, Navarro J, Fernandez-Yañez J, et al. Evaluation of an immunological score to assess the risk of severe infection in heart recipients. Transpl Infect Dis 2014;16:802-12.

9. Carbone J, Lanio N, Gallego A, et al. Simultaneous monitoring of cytomegalovirus-specific antibody and T-cell levels in seropositive heart transplant recipients. J Clin Immunol 2012;32:809-19.

10. Westall GP, Mifsud NA, Kotsimbos T. Linking CMV serostatus to episodes of CMV reactivation following lung transplantation by measuring CMV-specific CD8+ T-cell immunity. Am J Transplant 2008(8):1749-54.

11. Kotton CN, Kumar D, Caliendo AM, et al. Updated international consensus guidelines on the management of cytomegalovirus in solid- organ transplantation. Transplantation 2013;96:333-60. 
12. Sarmiento E, del Pozo N, Gallego A, et al. Decreased levels of serum complement C3 and natural killer cells add to the predictive value of total immunoglobulin $\mathrm{G}$ for severe infection in heart transplant recipients. Transpl Infect Dis 2012;14:526-39.

13. Sarmiento E, Lanio N, Gallego A, et al. Immune monitoring of anti cytomegalovirus antibodies and risk of cytomegalovirus disease in heart transplantation. Int Immunopharmacol 2009;9:649-52.

14. Gerna G, Lilleri D, Fornara C, et al. Differential kinetics of human cytomegalovirus load and antibody responses in primary infection of the immunocompetent and immunocompromised host. J Gen Virol 2015;96:360-9.

15. Sarmiento E, Rodríguez-Hernández C, Rodríguez-Molina J, et al. Impaired anti-pneumococcal polysaccharide antibody production and invasive pneumococcal infection following heart transplantation. Int Immunopharmacol 2006;6:2027-30.

16. Kumar D. Immunizations following solid-organ transplantation. Curr Opin Infect Dis 2014;27:329-35.

17. Yoshizumi T, Shirabe K, Ikegami T, et al. Decreased immunoglobulin G levels after living-donor liver transplantation is a risk factor for bacterial infection and sepsis. Transpl Infect Dis 2014;16:225-31.

18. Ohsumi A, Chen F, Yamada T, et al. Effect of hypogammaglobulinemia after lung transplantation: a single-institution study. Eur J Cardiothorac Surg 2014;45:e61-7.

19. Farmer DG, Kattan OM, Wozniak LJ, et al. Incidence, timing, and significance of early hypogammaglobulinemia after intestinal transplantation. Transplantation 2013;95:1154-9.

20. Chambers DC, Davies B, Mathews A, et al. Bronchiolitis obliterans syndrome, hypogammaglobulinemia, and infectious complications of lung transplantation. J Heart Lung Transplant 2013;32:36-43.

21. Fernández-Ruiz M, López-Medrano F, Varela-Peña P, et al. Monitoring of immunoglobulin levels identifies kidney transplant recipients at high risk of infection. Am J Transplant 2012;12:2763-73.

22. Kobashigawa JA, Kiyosaki KK, Patel JK, et al. Benefit of immune monitoring in heart transplant patients using ATP production in activated lymphocytes. J Heart LungTransplant 2010;29:504-8.

23. Gorbacheva V, Ayasoufi K, Fan R, et al. B cell activating factor (BAFF) and a proliferation inducing ligand (APRIL) mediate CD40-independent help by memory CD4 T cells. Am J Transplant 2015;15:346-57.

24. Ye Q, Wang L, Wells AD, et al. BAFF binding to T cell-expressed BAFF-R costimulates T cell proliferation and alloresponses. Eur J Immunol 2004;34:2750-9. 